\title{
Les débuts hésitants de la soviétisation de la littérature française à l'Université de Bucarest (1948-1949). Les programmes analytiques comme instrument de contrôle ${ }^{1}$ Dragoș JIPA
}

\begin{abstract}
Starting from previously unexplored primary sources, namely the syllabi of French literature developed for the first time at the University of Bucharest in 1948-1949, this article aims to illustrate the transformations of foreign literatures teaching in Romania at that time, in the context of the education reform decree adopted by the new Communist regime. In the field of French literature, which was an important tool in disseminating the official ideology, the analysis of these syllabi, both from the point of view of the authors, professors trained in the interwar period, such as Nicolae N. Condeescu (19041966), and of the pedagogical contents conveyed (literary works, writers, explanatory discourses), shows the internal mechanisms of the Sovietization process put into practice by the new regime. This process was not an immediate and mechanical imitation of the Soviet model, but a cultural transfer that depended on the actors of the educational process, their skills (more or less developed in terms of ideology and even in their command of Russian), as well as the previous institutional history of the university, particularly that of the department of French language and literature. Following the elaboration of the first versions of the syllabus of French literature, established by the new regime as tools of ideological control, we can capture the way in which their authors strove to apply the new Marxist discursive framework, but only in a formal manner, without developing a substantial reflection on French literature from this point of view.
\end{abstract}

Keywords: Sovietization, French literature, Syllabus, University of Bucharest, Nicolae N. Condeescu.

Pour le chercheur qui dépouille les archives de l'Université de Bucarest, les dossiers des années 1948 et 1949 sont différents de tout ce qu'il avait pu voir auparavant, concernant les périodes précédentes. Cette différence s'explique par le contexte politique particulier, l’année 1948 étant celle de la transformation du système d'enseignement roumain, conformément aux injonctions

1 Cet article a été réalisé dans le cadre du projet TRANSINTELL 21-AUF/01.03.2019 (« Transferts culturels et champs intellectuels transnationaux. Modernité et anti-modernité à l'Ouest et à l'Est: France, Roumanie, Serbie »), avec le soutien financier de l'AUF et de l'IFA. 
du décret 175/1948 promulgué par le nouveau régime communiste ${ }^{2}$. Au niveau universitaire, le changement vise le contrôle idéologique des institutions, ainsi que la réorganisation du système, censé dorénavant accomplir le programme du Parti Ouvrier Roumain. Les chercheurs qui ont étudié ces transformations ont mis en lumière la radicalité de la transformation mise en œuvre par le nouveau pouvoir, tant au niveau de la morphologie du corps enseignant (les épurations ${ }^{3}$, les nouveaux mécanismes de sélection ${ }^{4}$ ) qu’au niveau de l'introduction du marxisme dans les programmes d'enseignement de toutes les facultés ${ }^{5}$. Toutefois, par rapport aux documents produits dans le système universitaire roumain à partir du milieu du XIX ${ }^{\mathrm{e}}$ siècle, on voit apparaître dans les archives de l'institution un nouveau type de sources qui représentent une partie importante des dossiers de ces années-là ${ }^{6}:$ des « programmes analytiques » pour toutes les disciplines enseignées. Détaillés sur des centaines de pages, avec les contenus de chaque matière et le calendrier des enseignements (cours, pro-séminaires et séminaires), ces programmes expriment un état du savoir acquis et systématisé par les enseignants en vue de la transmission aux étudiants. Ce qui de nos jours est, pour les professeurs de l'université, au moins en Roumanie, un document obligatoire, exigé par les différents organismes d'assurance de la qualité de l'enseignement supérieur, était en 1948 une nouveauté, au moins pour ce niveau d'études.

2 Pendant l'année 1948, le processus de soviétisation commencé quelques années auparavant s'intensifie. Après le 23 août 1944, quand la Roumanie change de camp et commence à lutter contre l'Allemagne nazie aux côtés de l'Armée rouge, les communistes roumains avec l'aide des soviétiques imposent le gouvernement de Petru Groza en mars 1945, falsifient les résultats des élections du 9 novembre 1946, forcent la dissolution des autres partis pendant l'été 1947 et l'abdication du Roi le 30 décembre 1947. Au début de l'année 1948, la nouvelle République Populaire conclut le traité d'amitié avec l'Union Soviétique, légalise la répression de « l'activité contre-révolutionnaire » et adopte une nouvelle Constitution. Au mois de juin 1948, le régime nationalise les entreprises industrielles, financières, d'assurance et de transport et commence la transformation de l'agriculture et de l'enseignement.

3 Maria Someșan, Universitate și politică în deceniile 4-6 ale secolului XX - episoade și documente (Bucarest: Editura Universității din București, 2004), 241-276.

4 Alina Pavelescu, « Noile politici de selecție a studenților și a profesorilor de științe sociale la Universitatea din București, 1948-1955, I », Arhivele Totalitarismului 1-2 (2013) : 93-109.

5 Les formes et les effets de la soviétisation dans l'enseignement roumain ont été étudiés par Cristian Vasile dans « Sistemul de învățământ românesc în epoca stalinistă (1947-1953). Considerații », Revista Istorică, XX (2009) : 115-137. Sur les circonstances de l'adoption du décret 175, voir les pages 119-123.

6 Archives Nationales de la Roumanie - Succursale Municipale Bucarest (dorénavant SMBAN), Fonds Universitatea din București, Rectorat, dossiers 97/1948, 86/1949, 96/1949. 
Du point de vue de l'histoire des disciplines ${ }^{7}$, ces programmes d'enseignement constituent une source d'une grande valeur. Le savoir propre à un domaine, à un certain moment et dans un certain lieu, y est résumé dans ses composantes essentielles, ainsi que dans ses articulations, en fonction des différentes étapes de sa transmission pédagogique. Toutefois, ce type de document n'a pas une teneur uniquement scientifique. Dans tout régime politique moderne, que ce soit une démocratie libérale ou un régime autoritaire, les programmes véhiculent une certaine conception de la discipline en question, propre aux institutions qui les exigent, les élaborent et les mettent en œuvre. Par ces programmes, les autorités s'assurent que les élèves ou étudiants recevront une formation commune, conforme à ce qu'elles considèrent comme le contenu légitime de la discipline. Pierre Bourdieu le précise : « les programmes sont une affaire d'État ; changer un programme, c'est changer la structure de distribution du capital, c'est faire dépérir certaines formes de capital. La culture légitime est la culture de l'État. $\gg^{8}$ Cette dimension politique des programmes est d'autant plus visible au cours des périodes de passage d'un régime politique à un autre, comme c'est le cas de celle qui nous intéresse dans cette étude. En dépit de son caractère très général (beaucoup de détails devaient être précisés par des décisions du Ministère), le décret 175 adopté en août 1948 mentionnait à l'article 20 «l'élaboration de programmes analytiques minimaux, obligatoires et uniques pour toutes les chaires d'une même spécialité $\gg^{9}$. Vue à juste titre comme une composante de l'action politique du régime qui visait la limitation de l'autonomie universitaire par le contrôle central de l'enseignement ${ }^{10}$, l'élaboration de programmes correspondait toutefois à une pratique ancienne dans le système d'enseignement roumain, autant primaire que secondaire. Depuis les années 1860, le Ministère faisait publier périodiquement dans le Moniteur Officiel les programmes d'enseignement pour toutes les disciplines et pour toutes les classes, du niveau primaire jusqu'au lycée. Le savoir est déjà une affaire d'État, dès la création de la Roumanie moderne. L'importation du modèle soviétique consiste à généraliser cette pratique au niveau de l'enseignement supérieur, avec l'intention d'exercer un contrôle idéologique accru sur les contenus enseignés.

7 Parmi les études récentes sur la littérature française comme discipline scolaire, voir Clémence Cardon-Quint, Des lettres au français. Une discipline à l'heure de la démocratisation (19451981) (Rennes: Presses universitaires de Rennes, 2015).

8 Pierre Bourdieu, Sur l'État. Cours au Collège de France (1989-1992) (Paris: Raisons d'agir/Le Seuil, 2012), 162.

9 Gheorghe Bunescu (dir.), Antologia legilor învățământului din România (Bucarest: Institutul de Științe ale Educației, 2004), 343.

${ }^{10}$ Alina Pavelescu, « Noile politici de selecție $\gg, 99$. 
Ce type de sources peut ainsi constituer le point de départ d'une enquête visant à élucider les modalités de la transformation de l'enseignement supérieur en Roumanie, à l'époque de l'instauration du régime communiste. Les recherches sur la soviétisation des universités en Europe de l'Est ont pu découvrir comment le modèle original a été imposé dans différents contextes. L'exemple allemand ${ }^{11}$ montre comment les autorités soviétiques d'occupation essaient d'introduire ces programmes depuis 1946, mais sans grand succès : en 1949, les officiers soviétiques déplorent le fait que les universités « continuent d'enseigner des cours inutiles et nuisibles » et, en 1951, la Division Éducative de l'Administration Militaire Soviétique en Allemagne sollicite les programmes standard des universités de Moscou. D'autres recherches ${ }^{12}$ expliquent pourtant que, loin d'être un transfert culturel automatique et mécanique, la nature des transformations est déterminée par plusieurs facteurs propres aux pays d'accueil, entre autres la composition du corps professoral, les diverses modalités des « sorties de guerre » et, finalement, la culture politique de la communauté qui se situait du côté « récepteur » du transfert. De ce point de vue, l'étude des programmes analytiques que les universitaires roumains doivent élaborer pour la première fois en 1949 peut contribuer à une connaissance plus fine de ce processus, en mettant à profit deux tendances du champ de l'histoire des disciplines. Premièrement, si les disciplines sont vues comme des « créations [internes] du système scolaire $\gg\left(\right.$ A. Chervel $\left.{ }^{13}\right)$, l'étude des programmes peut montrer comment le paradigme marxiste s'articule aux savoirs déjà existants, comment il les transforme et oriente leur enseignement. Cette histoire « interne $\gg$, des contenus, doit toutefois être complétée par ce qu'on pourrait appeler une histoire « externe ». Ainsi, la seconde tendance voit les programmes non pas comme des « dispositifs consensuels et cohérents de transmission des connaissances $\gg$, mais comme le résultat d'interactions multiples, entre une pluralité d'acteurs, chacun aves ses stratégies et ses intérêts. Cette perspective, illustrée par Patricia Legris ${ }^{14}$ pour le cas de la discipline historique en France pendant la seconde moitié du $\mathrm{XX}^{\mathrm{e}}$ siècle, implique la prise en compte non seulement des processus d'élaboration des programmes, mais aussi des acteurs qui

${ }^{11}$ Voir Natalia Tsvetkova, Failure of American and Soviet Cultural Imperialism in German Universities, 1945-1990 (Leiden, Boston: Brill, 2013), 151.

${ }^{12}$ John Connelly, Captive University. The Sovietization of East German, Czech, and Polish Higher Education, 1945-1956 (Chapel Hill, London: University of North Carolina Press, 2000).

${ }^{13}$ André Chervel, « L'histoire des disciplines scolaires. Réflexions sur un domaine de recherche $\gg$, Histoire de l'éducation 38 (1988) : 59-119.

${ }^{14}$ Patricia Legris, Qui écrit les programmes d'histoire? (Grenoble : Presses universitaires de Grenoble, 2014). 
les produisent, avec leurs biographies et leur position dans le champ du savoir, ainsi que par rapport au champ politique. Dans les contextes de forte hétéronomie, comme celui de l'instauration du régime communiste en Roumanie, une telle approche est d'autant plus nécessaire.

La discipline que nous étudions dans ce qui suit est la littérature française ${ }^{15}$. Moins importante pour le régime que la littérature nationale roumaine et l'histoire, la littérature française avait de quoi intéresser les nouveaux dirigeants, pour plusieurs raisons. D'une part, comme littérature « étrangère », elle fait partie du système de discours sur «l'étranger » qui contribue à la consolidation de l'identité nationale dans les États de l'Europe moderne. En prenant encore une fois l'exemple de la France, la création des chaires de littératures étrangères pendant le XIX ${ }^{\mathrm{e}}$ siècle coïncide avec l'affirmation scolaire de la littérature française ${ }^{16}$. D'autre part, au $\mathrm{XX}^{\mathrm{e}}$ siècle, l'enseignement des langues et des littératures étrangères devient un instrument privilégié des diplomaties culturelles et traduit les relations entre les États. ${ }^{17}$ Même l'apparition en Europe d'une nouvelle discipline comme la « littérature comparée » avec la création de la Revue de littérature comparée au début des années 1920 n'est pas exempte des tentations nationalistes. ${ }^{18}$ En raison d'une certaine francophilie historique des élites roumaines, dès la fondation des premières deux universités dans les années 1860, à Iași et à Bucarest, les chaires de langue et littérature françaises apparaissent dans l'organisation institutionnelle des facultés, tandis que, jusqu'à la fin du XIX siècle, la littérature roumaine est enseignée dans le cadre d'une chaire intitulée « Histoire critique des Roumains et de la littérature roumaine $\gg$. Continuant une tradition qui remonte au XVIII ${ }^{\mathrm{e}}$ siècle, la langue française est très présente dans l'enseignement secondaire, jusqu'à devenir pendant l'entre-deux-guerres la langue étrangère obligatoire au lycée. En dépit de

${ }^{15}$ Même si en France on désigne par « lettres » les disciplines qui s'occupent de l'étude des littératures à l'école, nous avons préféré traduire les intitulés roumains des disciplines (《littératures étrangères », « littérature française »), parce qu'ils reflètent une spécificité nationale de l'institutionnalisation des études littéraires dans les facultés de philosophie et lettres (au sens large, incluant l'histoire, la psychologie, la sociologie, etc.) qui se transforment avec la mise en place du régime communiste en des facultés de philologie, de philosophie, d'histoire, etc.).

${ }^{16}$ Michel Espagne, Le paradigme de l'étranger : les chaires de littérature étrangère au XIX siècle (Paris : Éditions du Cerf, 1993).

${ }^{17}$ Voir, par exemple, Jérémie Dubois, L'enseignement de l'italien en France (1880-1940). Une discipline au cour des relations franco-italiennes (Grenoble : ELLUG, 2015).

${ }^{18}$ Fernand Baldensperger, «La littérature comparée : le mot et la chose, » Revue de littérature comparée, 1 (1921): 5-29. Pour une critique des présupposés nationalistes de «l'école française » de littérature comparée, voir René Wellek, « The Crisis of Comparative Literature, » dans Concepts of Criticism (New Haven and London : Yale University Press, 1963), 282-295. 
diverses critiques, venant de positions nationalistes (comme celles de Nicolae Iorga en 1906) ou de la fascination de la jeune génération pour les mouvements extrémistes (à partir des années 1920), la francophilie reste importante chez les anciennes élites roumaines surtout dans les années de l'instauration du régime communiste, au moins si on fait confiance aux rapports des diplomates français en poste à Bucarest jusqu'en $1948^{19}$. Par rapport aux autres littératures étrangères $^{20}$, le contrôle de la littérature française en tant que discipline représente ainsi un enjeu important dans le processus de transformation de l'enseignement imposé par le nouveau régime politique.

Considérer le programme d'enseignement de la littérature française comme un objet d'étude historique nous permet de formuler deux séries de questions. D'une part, qui sont les acteurs, individuels ou institutionnels, qui élaborent et mettent en œuvre cet enseignement et comment la « réforme » communiste se traduit-elle au niveau du corps enseignant de la discipline ? À travers ces questions, nous envisageons d'inscrire l'élaboration du programme dans une histoire de l'enseignement de cette littérature réalisé par des acteurs individuels, certes liés par leur appartenance à une même «communauté épistémique », mais qui doivent faire face à des conditions politiques changeantes. D'autre part, quelle représentation de la littérature française est véhiculée par ces programmes et comment se différencie-t-elle de celle qui était enseignée avant l'instauration du régime communiste? Dans ce qui suit, nous allons essayer d'apporter des éléments de réponse à ces deux séries de questions.

\section{Qui peut enseigner une littérature étrangère ? Trajectoires (trans)nationales, compétences professionnelles et allégeances politiques}

Si, jusqu'en 1948, la morphologie du champ universitaire roumain a une évolution assez régulière, la nouvelle législation introduite par le régime communiste change complètement les « règles du jeu ». D’une part, ont lieu des épurations massives du corps didactique. Déclenchées en 1945 contre les partisans du régime du maréchal Antonescu, elles visent des catégories de plus en plus larges : à commencer par ceux qui ont des relations scientifiques avec les universités allemandes et italiennes jusqu'à ceux qui n'ont pas de sympathies politiques de gauche. En 1947, des mises à la retraite et des suppressions de chaires contri-

${ }^{19}$ Annie Guénard-Maget, Une diplomatie culturelle dans les tensions internationales : La France en Europe centrale et orientale (1936-1940 / 1944-1951) (Bruxelles : Peter Lang, 2014).

${ }^{20}$ Les autres littératures étrangères enseignées à l'Université de Bucarest sont la littérature allemande, la littérature anglaise, la littérature italienne et la littérature russe. L’enseignement de la littérature espagnole s'arrête avec la retraite du professeur Alexandru Popescu-Telega en 1948. 
buent au changement du corps universitaire, à travers le remplacement des anciens professeurs par de nouvelles recrues qui adhèrent aux principes du Parti. Ainsi, en 1949, parmi les professeurs et les maîtres de conférences enseignant dans les facultés qui succèdent à l'ancienne faculté de philosophie et de lettres de Bucarest, seuls $7 \%$ à la faculté de philosophie, $14 \%$ à la faculté de philologie, $30 \%$ à la faculté d'histoire-géographie ont une ancienneté supérieure à 3 ans. ${ }^{21}$ En même temps, d'autres mécanismes de formation et de sélection du corps professoral sont mis en place pendant les décennies suivantes. L'adhésion à la politique du Parti devient le critère dominant pour l'entrée dans l'enseignement et la recherche qui se font à l'université (ou dans la nouvelle Académie de la République populaire roumaine).

Au niveau du corps professoral, l'impact de la réforme est diffèrent en fonction des disciplines. Si les sciences naturelles, les mathématiques, la physique ou la chimie ne présentent pas une importance si grande, les lettres, l'histoire, la philosophie sont beaucoup plus touchées, en vertu de leur capacité à transmettre l'idéologie officielle. Une demande de la Commission Alliée de Contrôle, l'organisme dirigé par les Soviétiques qui veille à l'application de l'armistice, en date du 7 juin 1946, annonce déjà ce qui va venir : la faculté est invitée à fournir d'urgence les cours d'histoire de la littérature roumaine, de grammaire du roumain et d'histoire de la langue roumaine. ${ }^{22}$

En comparaison à ces domaines, les « langues et littératures étrangères » représentent un enjeu particulier. Elles n'ont pas une dimension nationale explicite, sur laquelle le contrôle idéologique doit s'exercer, mais les enseignants titulaires sont vus comme les représentants des intérêts des pays étrangers, par rapport auxquels la Roumanie est en train de changer de politique. Ainsi, on enregistre des épurations (Alexandru Marcu), des transformations institutionnelles (la chaire d'histoire des États et de la culture des Slaves devient une chaire de langue et littérature russe) et, en général, une incertitude quant aux positions des titulaires de ces chaires.

En 1946, le conseil de faculté reconnaît la difficulté de trouver des spécialistes pour ces disciplines en Roumanie et recommande au Ministère d'embaucher par contrat des professeurs étrangers, « jusqu’à ce que, parmi les éléments roumains envoyés à l'étranger, se soient formés des chercheurs en mesure d'occuper avec dignité un poste de professeur d'université $\gg^{23}$. En ce qui concerne

${ }^{21}$ Maria Someșan, Universitate și politică, 264.

${ }^{22}$ SMBAN, Fonds Universitatea din București, Facultatea de Litere și Filosofie, Dossier 399/1946, f. 519.

${ }^{23}$ SMBAN, Fonds Universitatea din București, Facultatea de Litere și Filosofie, Dossier 406/1946-1948, f. 4. 
les professeurs français, cette pratique avait été inaugurée en 1919, avec la venue de la Mission Universitaire française en Roumanie ${ }^{24}$. Ainsi, on peut voir dans cette recommandation, conforme à la loi de 1943, un des derniers signes d'autonomie dans le recrutement dont les universités peuvent encore bénéficier, avant que la réforme communiste ne vienne restreindre sérieusement la possibilité pour un étranger d'enseigner le français en Roumanie ${ }^{25}$.

Parmi les « littératures étrangères », l'enseignement de la littérature française jouit d'un statut à part. D'une part, le français continue d'être la langue étrangère la plus demandée par les étudiants. Dans un rapport de décembre $1945^{26}$, le directeur de l'Institut Français de Bucarest, Jean Mouton, se félicite de « la proportion proprement écrasante des futurs étudiants en français, en face des autres disciplines de la philologie moderne $\gg$. C'est par l'intermédiaire du professeur Basil Munteanu, titulaire de la chaire de français à l'Université de Bucarest, que Mouton apprend « les constatations réconfortantes » sur « l'admiration des jeunes pour la France et sa vie spirituelle, leur foi dans l'avenir de ce pays, leur gratitude, leurs espérances $\gg$, telles qu'elles ressortent des réponses des candidats aux questions de l'examen d'admission. D'autre part, la diplomatie culturelle de la France est très présente en Roumanie, non seulement avec l'Institut Français des Hautes Études de Bucarest (avec nombre de cours et conférences), mais aussi avec un réseau important de Centres français à travers tout le pays, animés par quelques dizaines de professeurs appartenant à la Mission Universitaire qui enseignent dans les lycées et les facultés. La fin de la Guerre et la victoire des Alliés vont donner un nouvel élan tant aux étudiants qui souhaitent poursuivre l'étude de cette langue symbolisant pour eux la civilisation occidentale quaux autorités françaises qui désirent réaffirmer leur prestige dans les nouvelles « démocraties populaires ».

Toutefois, ce n'est plus Basil Munteanu qui répond aux intérêts des étudiants roumains, ainsi qu'à ceux de la diplomatie française. Ayant enseigné la littérature française à l’Université de Bucarest pendant les années difficiles de

${ }^{24}$ Sur les débuts de cette Mission, voir Ana-Maria Stan, « Vizita Misiunii Universitare Franceze în România (iunie 1919) și semnificațiile ei » dans Biserică, Societate, Identitate. In honorem Nicolae Bocșan, dir. Ion Cârja, Rudolf Gräf, Sorin Mitu, Ana-Victoria Sima (Cluj: Presa Universitară Clujeană, 2007), 691-697.

${ }^{25}$ L'exception est Henri Jacquier, professeur français membre de l'ancienne Mission Universitaire à Cluj, qui obtient un contrat de la part des autorités roumaines et décide de rester comme professeur à l'Université de Cluj. Ce choix va le séparer des autorités françaises pendant les années 1950 et la France lui refusera ses droits de retraite au début des années 1960.

${ }^{26}$ Centre des Archives Diplomatiques de Nantes (dorénavant CADN), Fonds Ambassade de France à Bucarest, 124PO/1, dossier 343, Note sur la proportion des étudiants de l'Université de Bucarest se consacrant à l'étude du français comme matière d'enseignement, 27 décembre 1945. 
la Guerre, ce spécialiste de littérature comparée formé pendant l'entre-deuxguerres à Paris auprès de Paul Hazard et de la Revue de littérature comparée décide d'y retourner lorsque l'occasion se présente (en 1946, comme directeur de l'École Roumaine de Fontenay-aux-Roses) et de ne plus rentrer en Roumanie.

Après son départ, il est remplacé à l'Université par Michel Dard. Ayant déjà travaillé comme lecteur de français pendant les années de la guerre, Dard est nommé pour remplacer Munteanu du 20 mars au 20 juin 1946, c'est-à-dire jusqu’à la fin de l'année académique. Mais le baron Dard n'est pas un simple lecteur de français. Sa fiche ${ }^{27}$ élaborée par les services secrets roumains révèle sa fonction d'ancien chef des renseignements français en Roumanie avant la Guerre et ensuite de directeur-adjoint de l'Institut Français pendant le mandat de Jean Mouton. Son activité montre à quel point l'enseignement de la littérature française en Roumanie a eu aussi une dimension politique pendant la guerre, à savoir la lutte contre le nazisme. D'une part, Dard est le responsable de l'organisation des cours publics et des conférences à l'Institut de Bucarest, une activité que la France développe à partir de 1941 pour contrecarrer la propagande allemande et italienne. ${ }^{28}$ D'autre part, avec Mouton et d'autres professeurs français de l'Institut, Dard fait partie d'un réseau secret qui recueille des informations sur les mouvements des troupes allemandes et les transmet aux Britanniques ${ }^{29}$. Ainsi s'expliquent les nombreuses notes que les agents des services secrets roumains écrivent au sujet de Dard et de ses conférences à l'Institut, lors desquelles il coordonnerait non seulement l'envoi de livres et de revues aux professeurs de français en Roumanie, mais aussi l'action contre Allemands, discutant le rôle des Français libres ou la contribution des colonies à la guerre.

Après le départ de Michel Dard, c'est George Oprescu, doyen de la faculté, qui est chargé d'occuper la chaire de français jusqu'au retour de son collègue. Deux documents des archives de la faculté, l'un daté le 2 décembre 1946 et l'autre le 20 mars 1947, font état de l'avis positif du Ministère pour cette suppléance. Pour l'historien de l'art roumain et directeur du Musée « Toma Stelian $\gg$ ce n'est pas sa première expérience de professeur de français. Ayant commencé sa carrière dans cette discipline avant la Première Guerre, à savoir dans les lycées de Giurgiu et de Turnu-Severin, il enseigne aussi dans la nou-

${ }^{27}$ Consiliul Naţional de Studiere a Arhivelor Securității (dorénavant CNSAS), Fonds Institutul Francez de Înalte Studii (1935-1946), D 0013417, f. 105. Voir aussi Ana-Maria Stan, « Aspects de la Résistance française en Roumanie après 1940. Diplomates, enseignants et écrivains » dans De part et d'autre du Danube. L'Allemagne, l'Autriche et les Balkans de 1815 à nos jours, dir. Mathieu Dubois, Renaud Meltz (Paris : PUPS, 2015), 139.

${ }^{28}$ Ibidem, f. 39.

${ }^{29}$ Dennis Deletant, British Clandestine Activities in Romania during the Second World War (Basingstoke: Palgrave Macmillan, 2016), 120. 
velle université roumaine « Ferdinand » de Cluj entre 1919 et 1923, avant son départ à la Société des Nations comme secrétaire de la Commission de Coopération Intellectuelle. L'autre argument qui détermine sans doute cette suppléance est le rapprochement de George Oprescu des milieux diplomatiques et universitaires français. Une chaire de civilisation française, financée par la France, avait été fondée pour lui à l'Université de Bucarest à la fin des années 1920 et l'idée est reprise en 1947 par le nouveau directeur de l'Institut Français, Philippe Rébeyrol. Cette fois, le premier - et l'unique - titulaire de la chaire, venu de France pendant un semestre, est l'historien Ernest Labrousse.

Les liens de longue date entre George Oprescu et l'Institut Français contribuent aussi à la participation d'autres professeurs français à l'enseignement de la discipline à l'Université de Bucarest. Embauchés par contrat sur la recommandation de Basil Munteanu, Robert Régnier commence son activité de lecteur le 17 novembre 1945 et Jean Sirinelli le 30 octobre 1946. Le premier, évadé d'un camp allemand de prisonniers, vient de Cluj, où il a été depuis 1943 lecteur à l'université hongroise et directeur du Centre Culturel Français. Jusqu'en 1948, parallèlement à son activité de lecteur de français à l'Université de Bucarest, il donne des cours à l'Institut Français, où il organise aussi des « matinées poétiques $\gg$ qui ont un grand succès chez le public bucarestois ${ }^{30}$. Le second, venu en Roumanie à l'automne 1946 pour remplacer Michel Dard, est aussi le responsable des conférences organisées par l'Institut Français tant à Bucarest que dans les autres villes ${ }^{31}$.

Cette participation importante des professeurs français à l'enseignement universitaire et secondaire roumain s'arrête de manière brusque en juin 1948 avec la résiliation de tous les contrats, ce qui sonne le glas de la Mission Universitaire française. Début juillet, l'Ambassadeur de France essaie en vain d'obtenir une dispense du Ministère roumain de l'Instruction pour les professeurs qui ont travaillé dans le supérieur, en employant tous les arguments possibles : l'absence de professeurs roumains ayant les titres nécessaires pour enseigner à l'université, la pratique de l'échange des lecteurs natifs qui fonctionne partout dans le monde, y compris dans les « démocraties populaires », le dévouement des enseignants français qui travaillent en Roumanie depuis des décennies, mais sans aucun succès ${ }^{32}$. Le mois d'août 1948 voit paraître le décret sur la réforme

${ }^{30} \mathrm{CADN}$, Fonds Ambassade de France à Bucarest, 124PO/1, dossier 346, Matinées poétiques organisées par l'Institut Français, 23 mai 1947.

${ }^{31} \mathrm{CADN}$, Fonds Ambassade de France à Bucarest, 124PO/1, dossier 346, Congrès de la Mission Universitaire Française, 17 juin 1947.

${ }^{32} \mathrm{CADN}$, Fonds Ambassade de France à Bucarest, 124PO/1, dossier 343, Résiliation des contrats des professeurs de la Mission Universitaire en Roumanie, 2 juillet 1948. 
de l'enseignement, qui transforme l'enseignement roumain selon le modèle soviétique et, quelques mois plus tard, la Roumanie dénonce l'accord culturel franco-roumain de 1939, ce qui entraîne la fermeture de l'Institut Français ${ }^{33}$. Si l'enseignement des littératures étrangères peut être vu comme un exemple classique de circulations transnationales entre les différents espaces universitaires, la suppression de la Mission Universitaire française par les autorités communistes illustre aussi les difficultés qui peuvent apparaître lorsque les différentes institutions ou États empêchent ces circulations et mettent en place des frontières nationales dures qui affectent les relations universitaires.

À l'Université de Bucarest, les départs de Régnier et de Sirinelli laissent cinq cents étudiants sans professeur. L'été 1948 est consacré à la recherche de remplaçants. Même si plusieurs sources (une lettre de Valentin Lipatti, frère cadet du fameux compositeur et doctorant en littérature française, adressée au professeur Tudor Vianu ${ }^{34}$, un message du directeur de l'Institut Français pour le Quai d'Orsay ${ }^{35}$ ) suggèrent que le professeur Nicolae Popa, titulaire de la chaire de français à l'Université de Iași serait le candidat avec les plus grandes chances, celui qui est finalement choisi, sur la recommandation des professeurs George Oprescu et Alexandru Rosetti ${ }^{36}$, est Nicolae N. Condeescu, à l'époque titulaire de la chaire de français à l'Académie commerciale de Brașov.

Né le 19 octobre 1904 (son père était fonctionnaire de préfecture), diplômé de la faculté de lettres et de philosophie de Bucarest en 1925, élève de l'École Normale Supérieure de la rue d'Ulm (promotion 1928), N. N. Condeescu obtient son doctorat ès lettres à Bucarest et enseigne dans différents lycées jusqu'en 1944, année de l'obtention du poste à Brașov. En juillet 1947, sans savoir ce que l'avenir lui réserve, il envoie une lettre à Basil Munteanu, à Paris :

Ici nous vivons tous sous le signe de l'épuration - massive et radicale cette fois - qu'on nous prépare. Si j’en suis atteint, je répéterai comme Virgile fortunatus et ille deos qui novit agrestes et j'installerai mes pénates à Pucioasa pour faire de la pomiculture. En exerçant n'importe quel autre métier, je m'en tirerai sans doute mieux que dans l'enseignement. Savez-vous que notre traitement actuel est d'environ 5 millions par mois, mais qu'il nous en faut au

${ }^{33}$ Pour plus de détails sur les circonstances de la fermeture de l'Institut Français, voir André Godin, Une passion roumaine, Histoire de l'Institut Français des Hautes Etudes en Roumanie (1924-1948) (Paris : L'Harmattan, 1997).

${ }^{34}$ Maria Alexandrescu Vianu, Vlad Alexandrescu (éd.), Scrisori către Tudor Vianu, II, (19361949) (Bucarest: Ed. Minerva, 1994), « Lettre de Valentin Lipatti », 9 août 1948, 322.

${ }^{35} \mathrm{CADN}$, Résiliation des contrats.

${ }^{36}$ SMBAN, Fonds Universitatea din București, Facultatea de Litere și Filosofie, Dossier 406/1946-1948, f. 64. 
moins trente pour vivre, non pas décemment, mais pour vivre tout court ? Une fois de retour, si tant est que vous commettrez la faute de rentrer avant que cette situation lamentable ait cessé, une fois de retour vous dis-je, vous regrettez votre Fontenay et vous prendrez vos heures actuelles de lassitude et de découragement pour du bon vieux temps $!^{{ }^{37}}$

Présentant apparemment des garanties intellectuelles, mais aussi idéologiques, étant membre du Parti depuis 1947 et membre de l'ARLUS ${ }^{38}$, Condeescu est nommé à la fin de 1948 professeur de français à l'Université de Bucarest. Condeescu voit probablement sa nomination comme une réussite, après de trop longues années passées dans l'enseignement secondaire et à un poste dans une académie commerciale qui ne correspondait guère à sa préparation de normalien. Une nomination qu'il n'aurait jamais obtenue dans les circonstances antérieures, lorsque Basil Munteanu était le titulaire de la chaire et que les professeurs français y travaillaient comme ses assistants. Les autres enseignants qui, sans avoir le grade de professeur, assurent cette année-là les séminaires pour les étudiants sont Valentin Lipatti, nouvellement recruté à l'Université, ainsi que Maria Pătrășcanu et Ana Pierret-Antoniu, déjà assistantes à la chaire de français. Condeescu est aussi le responsable de la nouvelle section de philologie moderne, qui comprend toutes les chaires de langues et littératures modernes, plus une chaire de littérature universelle dont le titulaire est Tudor Vianu, critique littéraire et ancien titulaire de la chaire d'esthétique, supprimée en $1948^{39}$.

Comme dans le cas des autres littératures étrangères ${ }^{40}$, l'année 1948 marque ainsi le renoncement aux professeurs étrangers qui sont vus comme des instruments des puissances occidentales ne pouvant être contrôlés par le régime. Leurs remplaçants roumains doivent toutefois, au moins à ce moment-là, faire preuve d'un niveau professionnel satisfaisant qu'ils pourront compenser dans les années suivantes par leur engagement politique. Il faut noter pourtant que la connaissance des littératures étrangères qui doivent être enseignées limite la restructuration du corps enseignant. De ce point de vue, le cas de la chaire de français n'est pas comparable à celui de la chaire de littérature roumaine, où

${ }^{37}$ Basil Munteanu, Corespondențe (Paris : Ethos, Ioan Cușa, 1979), 332.

${ }^{38}$ L'Association Roumaine pour le resserrement des Liens avec l'Union Soviétique (ARLUS) fut créée en 1944 et s'occupa de la propagande soviétique en Roumanie jusqu'au début des années 1960. Comprenant plusieurs sections, l'association fut dirigée par C. I. Parhon, médecin et professeur, président du Présidium de la Grande Assemblée Nationale de la République de 1948 à 1952.

${ }^{39}$ Le « cas » Tudor Vianu est discuté par Cristian Vasile, « Sistemul de învățământ », 123-126.

${ }^{40}$ Pendant la même période, les contrats des professeurs italiens ne sont pas renouvelés. 
l'historien de la littérature G. Călinescu est remplacé par le dentiste Ion Vitner ${ }^{41}$. D'autre part, cette observation ne vaut que pour le début de la soviétisation. L'historiographie récente relève l'illusion d'uniformité du régime : en fonction des circonstances internes (luttes pour le pouvoir) et externes (transformations au sein de l'U.R.S.S.), certains acteurs perdent leur position centrale et sont marginalisés, tandis que d'autres occupent le devant de la scène. Comme on le verra, le cas du professeur Condeescu illustre une trajectoire changeante.

\section{Planifier la littérature française : la matière et le processus}

En réponse à l'article du décret 175 , ainsi qu'aux multiples injonctions de l'administration de l'Université, N. N. Condeescu élabore en mars 1949 un ample document détaillant le programme du cours de langue et littérature françaises, pour la période entière des quatre années d'étude. Pour mieux faire comprendre ce que l'introduction des programmes analytiques a signifié pour la littérature française en tant que discipline universitaire, il faut d'abord passer en revue comment la matière était enseignée avant la mise en œuvre de ce décret.

Dès le milieu du XIX ${ }^{e}$ siècle, lorsque les premières universités roumaines sont créées et que la littérature française figure parmi les premières disciplines à se voir consacrer une chaire au sein des facultés de lettres et de philosophie, l'instance qui décide sur les contenus à transmettre aux étudiants est ostensiblement le professeur titulaire de la chaire. Influencés sans doute par la formation du professeur en question, par sa connaissance de l'état de la discipline en France, ainsi que par ses propres recherches, les cours de littérature française enseignés à l'université sont à l'image de leurs auteurs. Ainsi, Charles Drouhet, professeur à Bucarest entre 1915 et 1940, consacre ses cours annuels à des sujets aussi divers que « Le roman historique à l'époque du romantisme », « Le théâtre français au XVII ${ }^{e}$ siècle », « Les moralistes français », tout en réservant un deuxième cours à l'explication de textes d'auteurs tels Villon, Ronsard, Montaigne, La Fontaine, Boileau ou Voltaire ${ }^{42}$. Son successeur, Basil Munteanu, se concentre de son côté pendant la Seconde Guerre Mondiale sur la littérature des $\mathrm{XVII}^{\mathrm{e}}$ et XVIII $\mathrm{e}^{\mathrm{e}}$ siècles et leur applique l'histoire des idées qu'il chérit à l'instar

${ }^{41}$ Voir A. Berciu-Drăghicescu, Facultatea de Litere a Universității din București (Bucarest : Editura Universității din București, 2013).

${ }^{42}$ Pour les enseignements de Charles Drouhet à l'Université de Bucarest, nous nous permettons de renvoyer à notre article « La littérature française comme discipline académique dans la Roumanie de l'entre-deux-guerres. Le cas de Charles Drouhet » dans Veran Stanojevic, Milica Vinaver-Kovic (éd.) Les études françaises aujourd'hui 2018. Interactions dans les Sciences du Langages, Interactions disciplinaires dans les études littéraires (Belgrade : Faculté de Philologie de l’Université de Belgrade, 2019), 293-307. 
de son maître, Paul Hazard. Dans le contexte du développement de « l'histoire de la littérature » comme paradigme central de l'enseignement littéraire depuis la fin du XIX ${ }^{\mathrm{e}}$ siècle, que ce soit en France ou ailleurs, cette relative liberté du choix des sujets des cours a de quoi surprendre. Toutefois, il ne faut pas oublier la place importante que la littérature française occupe dans le curriculum roumain pendant la première moitié du $\mathrm{XX}^{\mathrm{e}}$ siècle, importance visible au niveau de l'enseignement secondaire : l'élève de lycée étudie tout au long de ses études l'histoire de la littérature française, à commencer par une vue d'ensemble en $\mathrm{V}^{\mathrm{e}}$ classe, suivie par trois séquences par ordre chronologique, jusqu'en VIII ${ }^{\mathrm{e}}$ classe du lycée). Disposant ainsi, au moins en théorie, d'une connaissance générale de la littérature française avant l'admission à la faculté, l'étudiant est prêt à recevoir un enseignement plus poussé, qui couvre des époques déterminées de la littérature française ou des écrivains précis.

Pendant l'année 1948, lorsque, suite au départ de Basil Munteanu, les enseignements sont assurés par les lecteurs français Jean Sirinelli et Robert Régnier, la matière est organisée ainsi ${ }^{43}$ : en $1^{\text {re }}$ année, le XVII ${ }^{\mathrm{e}}$ siècle, en $2^{\mathrm{e}}$ année, le $\mathrm{XVIII}^{\mathrm{e}}$ siècle, en $3^{\mathrm{e}}$ année, le XIX ${ }^{\mathrm{e}}$ siècle, et, en $4^{\mathrm{e}}$ année, à la fois le Moyen Âge, le $\mathrm{XVI}^{\mathrm{e}}$ siècle, la révision du XVII ${ }^{\mathrm{e}}$ siècle et des notions de littérature contemporaine. On peut reconnaître dans cette organisation du contenu l'influence de la conception de Munteanu, notamment dans l'enseignement par ordre chronologique ainsi que dans la distinction entre une approche générale et une perspective plus spécialisée : les « matières » des deux premières années sont communes à ceux qui optent pour le français comme matière principale et à ceux qui le choisissent comme matière secondaire, alors que les $3^{\mathrm{e}}$ et $4^{\mathrm{e}}$ années sont réservées à ceux qui ont le français comme matière principale.

Au début de l'année universitaire 1948-1949 qui commence au mois de décembre, conformément au décret entré en vigueur au mois d’août 1948, le nouveau titulaire de la chaire, le professeur N. N. Condeescu, fournit au nouveau doyen Iorgu Iordan le programme pour l'année en cours ${ }^{44}$. Le document comprend la description synthétique des cours et séminaires qu'il assure avec ses collègues, c'est-à-dire Valentin Lipatti, Maria Pătrășcanu et Ana Pierret-Antoniu. Les sujets des enseignements littéraires proviennent des recherches antérieures des professeurs : Condeescu enseigne «l'humanisme et la poésie au XVI ${ }^{\mathrm{e}}$ siècle $\gg$ et un cours général de présentation de la France (géographie, histoire, patrimoine). Lipatti dirige un séminaire sur «Molière et son théâtre » et un

${ }^{43}$ CADN, Fonds Bucarest, Institut Français des Hautes Études, 1919-1949, 126PO/1, Dossier 35, Rapport d'ensemble sur la situation de l'enseignement français, 14 mai 1948, 21.

${ }^{44}$ SMBAN, Fonds Universitatea din București, Rectorat, Dossier 97/1948, f. 169-173. 
autre sur « La comédie au XVIII siècle ». La description des thématiques révèle une vision traditionnelle de la littérature avec, par exemple, la conception platonicienne de Ronsard sur la majesté de la poésie et la mission du poète, agrémentée de sujets présentés dans le nouveau langage de l'idéologie en train de s'installer : L'Avare comme exemple de critique de la bourgeoisie et l'avarice comme étape du processus capitaliste dans la psychologie du bourgeois. D’autres séminaires, sous la direction d'Ana Pierret-Antoniu, sont consacrés à J.-J. Rousseau et Anatole France. À la différence de la situation antérieure, le programme de français offre également une série de cours de composition et d'explication de textes pour toutes les quatre années d'étude et, dans leur description, on peut identifier plusieurs éléments $d u$ «nouveau monde ». Ainsi, Maria Pătrășcanu propose aux étudiants des sujets de composition marqués par l'idéologie marxiste comme : «L'Idéologie du Moyen Âge a détruit le développement des forces productives de l'homme », «La religion catholique du Moyen Âge comme arme de défense de la classe dominante $»$, « Une interprétation moderne, progressiste du roman de Tristan et Iseut $\gg$. Ana Pierret-Antoniu propose, quant à elle, des explications de textes littéraires de la Résistance, en soulignant le rôle du Parti Communiste Français, avec des fragments de Benjamin Fondane, Louis Aragon, Paul Éluard, Jean Cassou, Robert Desnos, Pierre-Henri Simon, Vercors et Elsa Triolet, ainsi que, dans un autre séminaire, des « Actualités littéraires françaises », avec des articles extraits des revues Europe, La Pensée, Lettres françaises, etc. On pourrait conclure, à la lecture de ces programmes, que pendant l'année universitaire 1948-1949 et surtout au printemps 1949, l'enseignement de la littérature française était en grande partie l'œuvre des enseignants, libres dans le choix de leurs sujets, mais qui essayaient quand même de s'adapter aux nouvelles conditions politiques, en utilisant dans leurs présentations la perspective du matérialisme dialectique pour que les contenus conviennent aux nouveaux dirigeants. Dans un rapport envoyé fin juin 1949 en réponse aux questions du ministre Eduard Mezincescu sur la réussite de la réforme de l'enseignement, le doyen Iorgu Iordan répond que les programmes élaborés à l'automne 1948 n’ont pas pu être suivis en totalité parce que, d'une part, les procédures d'embauche des nouveaux professeurs se sont faites avec grand retard et, d'autre part, « leurs auteurs avaient l'habitude d'autrefois de concevoir le cours en fonction de leurs préférences personnelles et non pas dans l'intérêt de l'enseignement $\gg^{45}$.

Toutefois, alors que les enseignements mentionnés ci-dessus sont mis en application, la discussion institutionnelle autour des programmes s'intensifie. Au mois de janvier et de février 1949, le conseil de faculté invite les professeurs à

${ }^{45}$ SMBAN, Fonds Universitatea din București. Facultatea de Litere și Filosofie, Dossier 417/1949, f. 269. 
soumettre au secrétariat les programmes de leurs cours et, à partir du 15 février, les titulaires des cours présentent leurs programmes lors des réunions collectives des sections. Condeescu est nommé chef de la section de philologie moderne et préside en cette qualité les séances lors desquelles Tudor Vianu présente le programme du cours d'histoire de la littérature universelle (le 15 février), Bruno Colbert présente le cours d'histoire de la littérature allemande (les $1^{\text {er }}$ et 10 mars), Alexandru Balaci présente le cours d'histoire de la littérature italienne (le 8 avril $)^{46}$. Chargé aussi de la coordination des programmes, Condeescu élabore à son tour au mois de mars un autre programme de littérature française, qu'il présente pendant la réunion de la section le $1^{\mathrm{er}}$ avril. Ces réunions collectives constituent ainsi une première étape de la validation des programmes, avant d'être envoyés à la Direction des Études, l'instance de l'université chargée de vérifier la conformité idéologique des enseignements. D’ailleurs, le chef de la Direction, le physicien Valeriu Novacu, prodigue lors d'une des réunions de « précieuses indications » aux professeurs.

Dans plusieurs documents des archives on peut retrouver les principes formulés par Condeescu, qui sont à la base de l'élaboration de programmes unitaires pour les littératures étrangères. Ainsi, elles doivent être enseignées de manière chronologique et concordante, les cours de littérature doivent être complétés par des cours de civilisation et d'histoire de la langue, les exercices d'explication de texte doivent illustrer les cours de littérature, les cours et les séminaires doivent avoir le même nombre d'heures et la dénomination marxiste des époques doit être employée dans toutes les situations, tout comme la présentation de la littérature comme phénomène de superstructure. Cette planification des littératures étrangères, concomitante de celle de la littérature roumaine, représente la principale méthode de contrôle employée par les nouveaux dirigeants de l'université. Les programmes sont exigés par la Direction des Études du Rectorat qui vérifie la conformité avec les nouvelles injonctions du parti. Le rapport de fin d'année de la section de philologie moderne, daté du 17 mai 1949, révèle de nouveaux détails concernant le processus d'élaboration de ces programmes : les réunions collectives ont contribué à « éliminer l'explication idéaliste des phénomènes littéraires $\gg^{47}$, les cours pour la $4^{\mathrm{e}}$ année ont dû « combler les lacunes d'un passé caractérisé par le travail non planifié, lorsque les enseignements ne répondaient à d'autres critères qu'aux caprices du professeur $\gg$, mais Condeescu admet aussi des défauts :

${ }^{46} \mathrm{Il}$ n'y avait pas encore de professeur pour le cours de littérature russe, tandis que le cours de littérature anglaise était assuré par Mihnea Gheorghiu, qui venait d'être nommé professeur suppléant.

47 Cette citation et les suivantes, SMBAN, Fonds Universitatea din București. Rectorat, Dossier 86/1949, f. 151. 
Le manque de manuels soviétiques a rendu très difficiles les tentatives de restructuration de la matière, des traces involontaires de terminologie ou même d'interprétation idéaliste pouvant encore subsister dans les cours.

Les explications de textes [prévues dans les] programmes de langues et littératures étrangères se sont faites très difficilement parce que les étudiants ne trouvent plus dans le commerce les œuvres des grands écrivains français, allemands, italiens ou anglais.

Pour l'avenir, le chef de la section demande, entre autres, « un traducteur ou une équipe de traducteurs affectés à la faculté pour qu'on dispose au plus vite, en roumain, des principaux études et manuels soviétiques pour les domaines concernés » et suggère une liste d'ouvrages à traduire d'urgence, au cas où le Ministère de l'Enseignement ou les Éditions de l'État ont un tel projet.

Le programme de littérature française élaboré par Condeescu au printemps 1949 est précédé par une note qui en explique les principes :

La littérature française s'étudie de manière chronologique, évolutive; sa périodisation se fait selon les époques de développement des sociétés humaines établies par les grands penseurs du socialisme; en tant que phénomène de superstructure, la littérature d'une époque est précédée par une présentation de l'économie sociale de cette époque-là. ${ }^{48}$

L'histoire de la discipline est ainsi divisée en quatre périodes, correspondant aux quatre années d'études, présentées comme suit :

$1^{\text {ere }}$ année : l'époque féodale et le siècle de l'apparition du capitalisme (le Moyen Âge et la Renaissance);

$2^{e}$ année : la période d'ascension de la bourgeoisie sous la protection de l'absolutisme royal (le XVII e siècle, le classicisme);

$3^{\text {e }}$ année : la période de préparation de la Révolution bourgeoise de 1789 (le XVIII siècle);

$4^{\mathrm{e}}$ année : la période du triomphe du système capitaliste, avec sa période impérialiste et la lutte des classes entre le prolétariat et la bourgeoisie (depuis la Révolution bourgeoise de 1789 jusqu'à nos jours).

Organisée de cette manière, la littérature française est abordée à travers les mêmes écrivains qu'auparavant et on n'observe pour la plupart des périodes aucune élimination ou censure. La seule nouveauté, à l'exception de la stricte chronologie, est le cadre discursif qui indique le changement de régime ${ }^{49}$.

${ }^{48}$ Cette citation et la suivante, Ibidem, f. 37

${ }^{49}$ Cette adoption au niveau superficiel du nouveau cadre discursif marxiste, sans une réflexion substantielle sur la littérature française de cette perspective n'est pas sans rappeler les observations d'Alexei Yurchak sur la dimension performative des discours officiels dans l'Union Soviétique poststalinienne, discours dont la reproduction formelle était plus importante que 
Pour le traitement de chaque époque, si on laisse de côté les premières et les dernières phrases, telles « La Renaissance et l'Humanisme comme manifestations de l'ascension de la bourgeoisie et comme instruments de la lutte de classe contre la noblesse féodale », la liste des écrivains et des œuvres enseignés ne semble pas avoir été affectée par le nouveau contexte. Pour le XVII ${ }^{e}$ siècle, on y retrouve, aux côtés des grands classiques comme Corneille, Racine et Molière, les noms de Pascal, Bossuet, Madame de La Fayette, Fénelon, etc. De la même façon, pour le séminaire, on retrouve Théophile de Viau et Guez de Balzac parmi les auteurs des textes traités mais aussi Les Pensées de Pascal, Le Cid, Horace et Polyeucte de Corneille. Le dernier cours offre, apres une présentation détaillée de la littérature du XIX ${ }^{\mathrm{e}}$ siecle, à savoir Chateaubriand, Lamartine, Vigny, Hugo, Musset, Stendhal, Balzac, Flaubert, Zola, le symbolisme, une incursion dans ce qu'on pourrait appeler la littérature récente ou « contemporaine ». C'est ici qu'on peut voir le critère politique dans la sélection des auteurs : y figurent Romain Rolland, le roman populaire de l'entre-deux-guerres et la littérature de la Résistance (surtout Aragon et Éluard).

\section{Conclusions}

L'analyse des sources montre que, dans les premières années du régime, plus que le contenu proprement dit des programmes, c'est plutôt le processus complexe d'élaboration et de validation institutionnelle qui est le principal instrument de contrôle dans la soviétisation de la littérature française. À la différence du passé, quand le professeur, titulaire de la chaire, avait une grande autonomie dans l'enseignement de sa matière et que le seul compte rendu qu'il devait donner de son enseignement à l'université était un rapport de fin d'année, le nouveau régime politique qui se met en place après l'abdication du Roi impose un contrôle suivi, en exigeant l'élaboration de programmes selon la doctrine du parti, programmes qui soient chronologiques et uniformes dans leur présentation des littératures étrangères. Cette restructuration est faite toutefois par quelqu'un qui n’a pas dans sa biographie jusqu'à ce moment-là des épisodes d'engagement politique à gauche. Produit d'un parcours universitaire traditionnel, boursier roumain dans une grande école française, N. N. Condeescu ne voit pas la littérature française de la perspective du matérialisme historique ni même d'un point de vue marxiste, comme c'est le cas, par exemple, d'un Paul Bénichou qui publie en 1948 un ouvrage fondamental sur la littérature française du XVII ${ }^{\mathrm{e}}$ siècle, ${ }^{50}$ dans lequel il prend en compte les conditions sociales des

les contenus. Voir Alexei Yurchak, Everything Was Forever, Until It Was No More. The Last Soviet Generation (Princeton: Princeton University Press, 2005).

${ }^{50}$ Paul Bénichou, Morales du Grand Siècle (Paris : Gallimard, 1948). 
œuvres littéraires, conditions qui n'étaient pas discutées par « la critique bourgeoise du $\mathrm{XIX}^{\mathrm{e}}$ siècle $\gg$. Le marxisme de celui-ci est toutefois très personnel et met l'accent plutôt sur le côté éthique que sur les seules réalités économiques et sociologiques. ${ }^{51}$ À comparer ces deux perspectives, bien que dans un cas il s'agisse d'un programme analytique et dans l'autre d'un ouvrage plus ample, on ne peut s'empêcher d'observer le caractère assez caricatural de la lecture matérialiste de la littérature française par N. N. Condeescu. Comme dans le cas de la discipline historique ${ }^{52}$, l'adoption de la perspective marxiste est superficielle et simpliste, l'imposition de la chronologie canonique et de l'opposition progressiste-réactionnaire ne pouvant pas changer en profondeur le tableau de la littérature française.

D’ailleurs, au mois de mai 1950, Condeescu est exclu du Parti, dans le contexte des purges visant la soi-disant « déviation de droite ». Il continue d'enseigner à la chaire de français et, dans une biographie ${ }^{53}$ élaborée par le Service des Cadres de l'Université le 28 février 1961, il intervient pour démonter les causes de son exclusion qu'étaient la fortune de son père, son socialisme de droite et le fait d'avoir enseigné le français au Roi Michel entre 1934-1936 : son père n'aurait pas eu une vraie exploitation foncière, son socialisme serait venu du fait qu'il avait fréquenté le cercle des étudiants socialistes de l'ENS dans les années 1920 et il n'aurait pas été le professeur officiel du Roi, les quelques leçons données pendant 18 mois ne suffisant pas pour lui conférer ce titre. Un autre document de $1955^{54}$, toujours en provenance du Service des Cadres, le cite comme membre du parti fasciste d'A. C. Cuza de 1923 à 1928 (la raison de son exclusion en 1950) et le caractérise comme ayant « un niveau idéologique assez bas, des réminiscences petites-bourgeoises et des concepts anciens. Ses cours sont encombrés d'objectivisme et bourrés de fautes cosmopolites $\gg^{55}$. Selon la caractérisation d'une collègue, les cours donnés par Condeescu entre 1949 et 1950 étaient «pleins de détails, d'une érudition stérile, une mise à jour maladroite et idéalisme $\gg^{56}$. Ces documents sont réunis au début des années 1960, lorsqu'il souhaite réintégrer

\footnotetext{
${ }^{51}$ Sur l'évolution de Paul Bénichou du marxisme antistalinien des années 1930 aux positions antimarxistes des années 1970, voir Alexandre de Vitry, « Politique de l'histoire des idées : autour de Paul Bénichou » dans Histoire et historiens des idées : Figures, méthodes, problèmes, dir. David Simonetta, Alexandre de Vitry (Paris : Collège de France, 2020), 167-184.

52 Pour une synthèse sur l'évolution de l'historiographie roumaine, voir Bogdan Murgescu, A fi istoric în anul 2000 (Bucarest: All Educațional, 2000).

${ }^{53}$ CNSAS, Fonds Documentaire, I 0392183, f. 8.

${ }^{54}$ Ibidem, f. 23.

${ }^{55}$ Ibidem, f.16.

${ }^{56}$ Ibidem, f. 16.
} 
le Parti et qu'il fait l'objet d'une surveillance de la Securitate. Plusieurs notes fournies par ses collègues soulignent le fait que, depuis le temps de 1949, lorsque son niveau idéologique était assez faible, « on a pu noter une évolution évidente dans sa manière de voir les phénomènes de la littérature française, dans le sens d'une appropriation des principes de base de l'interprétation marxiste $\gg^{57}$. Chef du département de français et doyen de la nouvelle faculté de langues et littératures romanes, il réussit à garder l'équilibre entre les différents groupes d'enseignants, en se présentant comme très fidèle à la cause du Parti, jusqu'à sa mort, en 1966. Si dans la première partie de sa carrière ses recherches sont consacrées à des sujets de littérature comparée ${ }^{58}$, après l'instauration du régime communiste il s'impliquera dans les projets de la nouvelle section de traducteurs de l'Union des écrivains, en contribuant avec des préfaces et des notes aux traductions de Molière, La Fontaine, La Bruyère, Voltaire, Balzac, Victor Hugo, etc. À lire ces préfaces, on constate, comme dans le cas des programmes, le même usage du principe de la lutte des classes pour expliquer l'évolution de la littérature, sans pour autant oublier l'individualité des grands écrivains, leurs tempéraments et leurs préférences personnelles. Après sa mort, ses cours d'introduction à la philologie et d'histoire de la langue française seront publiés par ses collaborateurs. La soviétisation de la littérature française commencée sous sa direction en 1949 était en fait l'œuvre d'une personne sans trop de convictions de gauche, récemment convertie au nouveau régime et c'est pour cette raison que le processus en question commence de manière assez hésitante. Des recherches supplémentaires sont nécessaires pour approfondir la connaissance de la soviétisation, de sa temporalité et des négociations entre les divers acteurs. Ce qui apparaît à première vue comme une reproduction fidele du modèle soviétique d'interprétation de la littérature se révèle être un processus bien plus complexe ${ }^{59}$. Pour revenir aux programmes, une question nécessaire est celle du rapport entre le docu-

${ }^{57}$ Ibidem, f. 43.

${ }^{58}$ Voir N. N. Condeescu, La légende de Geneviève de Brabant et ses versions roumaines (Moniteur Officiel, Bucarest : 1938).

${ }^{59}$ Dans un rapport confidentiel de 1959 au Ministre de France à Bucarest, Henri Jacquier, professeur français à Cluj, explique ses diverses manières de contourner le discours officiel en employant des éléments du même discours : « Nous nous sommes fait tout un arsenal d'arguments protecteurs, tirés soit des maîtres du marxisme, soit des critiques soviétiques d'esprit plus indépendant, moins dogmatiques, à l'aide desquels nous nous gardons du 'sociologisme vulgaire' et de toutes les autres erreurs de méthode ou d'idéologie. Une seule formule comme celle selon laquelle 'le marxisme est ennemi de tout dogmatisme' suffit à nous assurer la même liberté que celle dont jouissent mes collègues en France. »CADN, Fonds Ambassade de France à Bucarest, 124PO/1, dossier 401. 
ment administratif, approuvé par les instances officielles de l'université, et la pratique effective de l'enseignement, dans l'amphithéâtre, devant les étudiants. Trouver une réponse à cette question est plus difficile, compte tenu des sources nécessaires pour trouver ce genre d'informations. L'exemple de Tudor Vianu, dont le cours de littérature universelle est critiqué dans un rapport de 1952 de la Section de Propagande du Parti pour « une conception objectiviste et cosmopolite $\gg^{60}$, peut fournir quelques pistes en ce sens.

D’ailleurs, au début de l'année universitaire 1949-1950, le 27 octobre, le doyen de la faculté de philologie demande au recteur de faire retirer le programme analytique élaboré par Condeescu et de le remplacer par un nouveau programme, élaboré par Theodosia Ioachimescu-Graur (la collègue qui critiquera plus tard son «idéalisme et érudition stérile »), récemment nommée maîtresse de conférences auprès de la chaire de langue et littérature française. Bénéficiaire elle-aussi d'une bourse en France dans les années 1920 et titulaire d'un doctorat obtenu à la Sorbonne en 1929 sur Amadis Jamyn, un disciple de Ronsard, l'ancienne épouse du linguiste aux convictions de gauche Alexandru Graur vient de finir un mandat de conseillère de presse à la légation roumaine de Paris. Pendant ce mandat, elle avait été affectée (faisant l'objet de mauvais traitements de la part de la police française, selon le Ministère roumain des Affaires Étrangères ${ }^{61}$ ) par les tensions qui avaient conduit entre été et automne 1948 à la rupture des relations culturelles entre la France et la Roumanie, symbolisée par la fermeture de l'Institut Français des Hautes Études de Bucarest. Le $1^{\text {er }}$ novembre 1948, le Ministère des Affaires Étrangères, dirigé alors par Ana Pauker, fait cesser sa fonction de conseiller de presse, vu sa nomination au Ministère de l'Enseignement Public. Les dirigeants communistes sentaient déjà, peut-être, le besoin de recrues plus convaincues pour la soviétisation de la littérature française à Bucarest.

\section{Rezumat}

Pornind de la niște surse inedite, programele analitice de literatură franceză elaborate pentru prima dată la Universitatea din București în anii 19481949, articolul de față își propune să ilustreze transformările din predarea literaturilor străine în România în acea perioadă, în contextul decretului de reformă a învățământului adoptat de noul regim comunist. În domeniul predării literaturii franceze, care avea un potențial important de difuzare a ideologiei oficiale, analiza acestor programe, atât din punctul de vedere

${ }^{60}$ Voir Cristian Vasile, « Sistemul de învățământ », 124.

${ }^{61}$ Archives du Ministère Roumain des Affaires Etrangères (AMAE), Franța, Dossier 43A/20/1948, f. 1 . 
al autorilor, profesori formați în perioada interbelică, precum Nicolae N. Condeescu (1904-1966), cât și din cel al conținuturilor pedagogice vehiculate (opere literare, scriitori, discursuri explicative), arată mecanismele interne ale procesului de sovietizare pus în practică de noul regim. Acest proces nu a reprezentat o imitare imediată și mecanică a modelului sovietic, ci un transfer cultural care a depins de actorii procesului de învățământ, de competențele acestora (mai mult sau mai puțin dezvoltate în ce privește ideologia ce trebuia aplicată sau chiar în privința cunoașterii limbii ruse), precum și de istoria anterioară a instituției universitare (în speță, a catedrei de limba și literatura franceză). Urmărind elaborarea primelor versiuni ale programelor analitice de literatură franceză, instituite de noul regim ca instrumente de control ideologic, putem surprinde modul în care autorii lor se străduiesc să aplice noul cadru discursiv marxist, dar nu reușesc să o facă decât la nivel formal, fără să dezvolte o reflecție substanțială din acest punct de vedere asupra literaturii franceze.

Cuvinte-cheie: sovietizare, literatură franceză, programe analitice, Universitatea din București, Nicolae N. Condeescu.

Dragoș Jipa, Department of French Language and Literature at the University of Bucharest. Email: dragos.jipa@g.unibuc.ro 